[Bull. Agr. Chem. Soc. Japan, Vol. 22, No. 4, p. 249 255, 1958]

\title{
Chemical Studies on the Autolysis of Meats
}

\section{Part VII. On the Chemical Changes of Myosin B during aging of Meats.}

\author{
By Masao Fujimaki and Nobuhiko Arakawa \\ Department of Agricultural Chemistry, Faculty of Agriculture, University of Tokyo
}

Received April 4, 1958

\begin{abstract}
In order to study whether or not myosin B has been altered during the aging of meats, experiments were carried out on salting out, viscosity, ATP-sensitivity, and ATPase of myosin $B$ solution.

Chemical properties of myosin $\mathbf{B}$ were found to be altered during the aging process of meat, especially AMS, a member of myosin B (thus named by one of authors) resulted in a high ratio of myosin in its constitution, and seemed to come to resemble the properties of myosin during the process.
\end{abstract}

In previous reports, ${ }^{1,2)}$ myosin, actin, myosin $B$, and sarcoplasmic proteins were determined quantitatively during the aging of meats, from which it was shown that the difference in the chemical natures of myosin-, and myosin BATPase during the aging process is dependent on the chemical changes of these proteins during the process.

As intact myosin B still remained in meats even after the meats had undergone the maximum of rigor mortis, and moreover the chemical nature of myosin B-ATPase has been altered during the aging process, it was interesting to study on the chemical natures of myosin B isolated from meats during the aging process to elucidate the mechanisms of meat tenderness subsequent to rigor motis.

In this report, salting out patterns, viscosity changes, and the effect of added adenosine triphosphate (ATP) on viscosity, i.e., ATPsensitivity, of myosin $B$ from meats during the aging process are presented.

\section{EXPERIMENTAL}

Methods. Preparation of myosin B from meat materials: Two kinds of myosin B, AMS and AML, 1) M. Fujimaki and Y. Nakajima, J. Agr. Cbem. Soc. Japan (in press).

2) M. Fujimaki, J. Agr. Chem. Soc. Japan (in press) . given such names by one of the authors were prepared in the same manner as previously reported ${ }^{1}$ from the following meats during the aging process (at about $0^{\circ}$ for 12 days) : rabbit (variety, Japanese white, body weight $3 \mathrm{~kg}$, female, longissimus dorsi), veal (variety, Holstein, one year old, female, part-neck muscle), cow (Japanese Black Breed of Cattle, seven years old, female, part-neck muscle), and horse meat (variety, unknown, eight years old, castrated, partdiaphram).

The name AMS was given to myosin B which is obtained by extracting meats for a short period with $0.6 \mathrm{M}$ potassium chloride solution and then precipitated by dilution to an ionic strength of 0.1 with glass redistilled water ("dilution method"). AML is another kind of myosin B, extracted with the Weber-Edsall solution, for an extended period from the residue remaining after extraction of AMS, and refined by the "dilution method" as described above.

Salting out: The following experiments on salting out of myosin B were carried out according to the procedure of Yagi et al. ${ }^{3)}$ An amount of one-tenth to two gram of ammonium sulfate was added to each $5 \mathrm{ml}$. of myosin B solution in $0.6 \mathrm{M}$ potassium chloride $(1 \mathrm{gN} / \mathrm{l})$, and after being allowed to stand over night at $0^{2}$, each solution was centrifuged and then filtered (Toyo filter paper, No. 3C). Optical density at $275 \mathrm{~m} \mu$ of each filtrate was measured with a Beckman model D.U. spectrophotometer.

3) K. Yagi et al., presented at the IX th Symposium on Enzyme Chemistry (1956). 
Viscosity : Viscosity of the myosin B solution in potassium chloride was measured at $1^{\circ}$ with an Ostwald viscosimeter. The intrinsic viscosity $[\eta]$ was calculated from the relative viscosity $\eta_{\text {rel }}$ as follows :

$$
[\eta]=\left(\eta_{\mathrm{rel}}-1\right) / C, \quad C=\operatorname{myosin} \mathrm{B} \mathrm{g} / \mathrm{l}
$$

ATP-sensitivity : To $5 \mathrm{ml}$. of myosin B solution in potassium chloride were added $0.25 \mathrm{ml}$ of $2 \times 10^{-2} \mathrm{M}$ magnesium chloride solution and $0.5 \mathrm{ml}$ of $1 \%$ neutral sodium ATP solution. After it was mixed thoroughly, the relative viscosity $\eta_{\text {rel }}(A T P$, was determined.

The ATP-sensitivity was calculated from the following equation.*

$$
\begin{aligned}
\text { ATP-sensitivity }= & \left(\log \eta_{\mathrm{rel}}-\log \eta_{\mathrm{rel}(\mathrm{ATP})}\right) / \\
& \log \eta_{\mathrm{rel}}(\mathrm{ATP}) \cdot 100 \%
\end{aligned}
$$

ATPase-activity : Activity of ATPase was measured as previously reported, 2 and the reaction mixture which contained a final-volume of $3.0 \mathrm{ml}$, composed of $1.0 \mathrm{ml}$ of $0.04 \mathrm{M}$ veronal or $0.1 \mathrm{M}$ citrate buffer solution, $0.5 \mathrm{ml}$
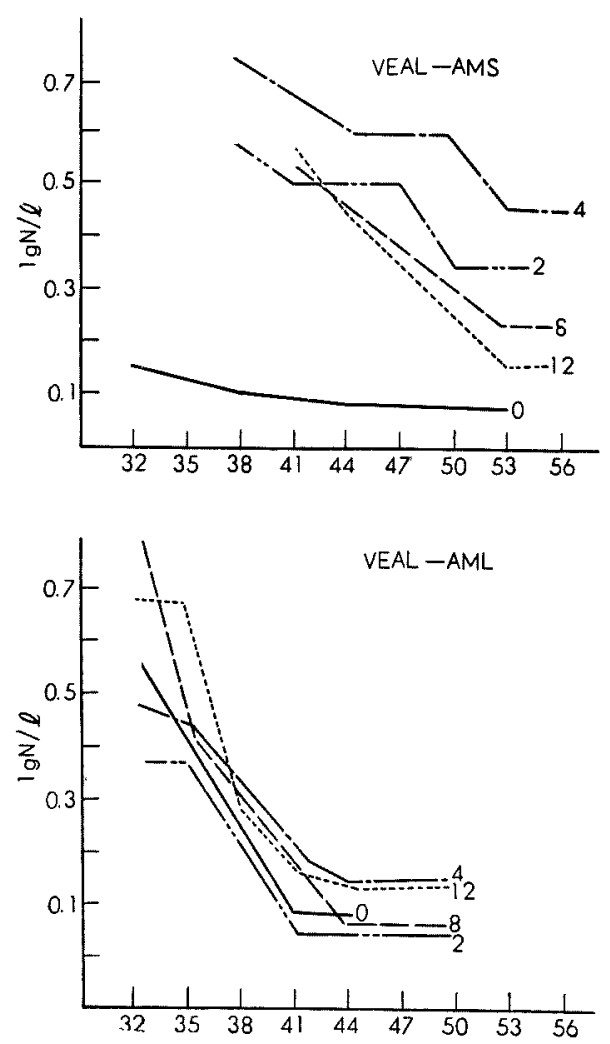

Saturation degree of ammonium sulfate in \%.

FIG. 1. Salting out Curves of Veal Myosin B Solution. Numbers in Fig. show days of aging.

4) W. Hasselback and G. Schneider, Biocbem. Z., 321, 462 (1951). of $6 \times 10^{-2} \mathrm{M} \mathrm{CaCl}_{2}, 0.5 \mathrm{ml}$ of $1.2 \mathrm{M} \mathrm{KCl}, 0.5 \mathrm{ml}$ of myosin $\mathrm{B}$ solution in potassium chloride and $0.5 \mathrm{ml}$ of $5 \times 10^{-3} \mathrm{M}$ neutral sodium ATP solution was incubated at $25^{\circ}$. At measured intervals of time, to aliquotes of the reaction mixture was added $1.0 \mathrm{mI}$ of $10 \%$ trichloroacetic acid solution and the protein precipiated was filtered off through a dry filter paper. The amount of inorganic phosphorus liberated in $1 \mathrm{ml}$ of the filtrate was determined colorimetrically using a modification of the Allen method.5)

One unit of ATPase was defined as the amount of enzyme which liberate $1 \gamma$ of inorganic phosphorus under the above conditions.

\section{RESULTS AND DISCUSSION}

Salting out: The results on salting out of myosin B solution are presented in Figs. 1 and 2. As shown in these figures, the optical densities of the AMS solutions from veal and



Saturation degree of ammonium sulfate in $\%$.

FIG. 2. Salting out Curves of Horse Meat Myosin B Solution.

Numbers in Fig. show days of aging.

5) M. Nakamura, J. Agr. Chem. Soc. Japan, 24, 1 (1950). 
horse meat obtained immediately after slaughter were very small as compared with those of AMS solutions from aged meats.

As the concentration of ammonium sulfate in the solution increased, salting out curves of AMS declined and turned to show a flat line.

The flat region was obtained by 38-41\% saturated ammonium sulfate with veal AMS immediately after slaughter, by $50 \%$ after 2 days, and by $53 \%$ after 4 to 12 days of aging.

As it may be seen the concentration of ammonium sulfate in the salting out of AMS has been altered with the aging of meats, the maximum precipitating region of AMS seemed to approach that of myosin.

In veal AML, the maximum precipitating region was obtained by $41 \%$ saturated ammonium sulfate, immediately after slaughter and the following 2 days, by $44 \%$ after 4 to 12 days of aging. As observed in the case with AMS, the concentration of ammonium sulfate in salting out of AML has been shifted toward higher concentrations with the aging of meat.

Similar results were obtained with the salting out of myosin B of horse meat as it is seen in Fig. 2.

These results, in which the concentration of ammonium sulfate in the maximum precipitating region of myosin $B$ has been raised with the aging of meats, seem to show changes of constitutional structure in myosin B with aging. For example, AMS from unaged meat seems to be different from the AMS of meats aged for 4-12 days, and AMS has a maximum precipitating region at a higher concentration of ammonium sulfate than AML. Therefore, from the results on salting out of myosin B, chemical differences of two kinds of myosin $\mathrm{B}$ will be obvious.

Viscosity : Intrinsic viscosity of myosin B in potassium chloride solution prepared from rabbit, veal, cow, or horse meat is presented in Table I. From the table, it is obvious that the intrinsic viscosity of AMS is lower than that of AML, irrespective of the kind of meats tested.
TABLE I

INTRINSIC VISCOSITY OF MYOSIN B SOLUTION.

\begin{tabular}{|c|c|c|c|}
\hline $\begin{array}{l}\text { Kinds of animal } \\
\text { meats }\end{array}$ & $\begin{array}{l}\text { ging period } \\
\text { (Days) }\end{array}$ & $\begin{array}{l}\text { Intrinsic } \\
\text { AMS }\end{array}$ & $\begin{array}{r}\text { Viscosity } \\
\text { AML }\end{array}$ \\
\hline \multirow{4}{*}{ Rabbit } & 0 & 0.27 & 0.50 \\
\hline & 4 & 0.43 & 0.98 \\
\hline & 8 & 0.17 & 0.60 \\
\hline & 0 & 0.19 & 0.35 \\
\hline \multirow{4}{*}{ Veal (Holstein Kind) } & 2 & 0.13 & 0.72 \\
\hline & 4 & 0.13 & 0.39 \\
\hline & 8 & 0.14 & 0.51 \\
\hline & $\mathrm{I} 2$ & 0.13 & 0.62 \\
\hline $\begin{array}{l}\text { Veal (Japanese Black } \\
\text { Breed of Cattle) }\end{array}$ & 0 & 0.21 & 0.46 \\
\hline & 0 & 0.06 & 0.29 \\
\hline \multirow{3}{*}{ Cow meat } & 2 & 0.04 & 0.28 \\
\hline & 4 & 0.08 & 0.39 \\
\hline & 8 & 0.06 & 0.37 \\
\hline \multirow{4}{*}{ Horse meat } & 0 & 0.26 & 0.45 \\
\hline & 4 & 0.24 & 0.44 \\
\hline & 8 & 0.32 & 0.54 \\
\hline & 12 & 0.18 & 0.42 \\
\hline
\end{tabular}

The intrinsic viscosities of AMS and AML from rabbit gave maximum values at about 4 days of aging, that is, at the time of maximum rigor. Similar results were obtained in the changes of intrinsic viscosities of horsemyosin B solution and the AML of veal and cow meats. These changes occurring in intrinsic viscosity of myosin B during the aging process of meats are therefore considered to indicate changes of constitutional structure in myosin B proceeding with the aging of meats and have some influence upon rigor mortis and the rigor off of meats.

On the other hand, the intrinsic viscosity of myosin B solution from different kinds of animal meats is presented in Table I from which is is observed that the intrinsic viscosity of myosin B from rabbit is very high, and that from the cow meat very low. Consequently, the difference of water binding capacity of meat depending on the kinds of animals may be partly attributed to the differences of intrinstic viscosity.

Also, from reports that the intrinsic viscosity of actomyosin (equivalent to myosin B referred in this paper) solution from fish muscles 
(Yellow-striped rockfish) decreases after one day of aging at $0^{\circ},{ }^{6)}$ chemical changes in muscles of fish post mortis are supposed to proceed more rapidly than in animal meat.

ATP-sensitivity : In aged meat it is important to determine the content of actomyosin (myosin B) and the ratio of myosin to actin in actomyosin. ${ }^{4)}$ The intrinsic or specific viscosity of myosin $\mathrm{B}$ is dependent on the relative ratio of actin to myosin in actomyosin, and when the ratio was $1: 2.5$, specific viscosity was at its highest. ${ }^{7)}$ When ATP is added to myosin $\mathrm{B}$ in potassium chloride solution at an appropriate concentration, viscosity decreases, the depression being maximum when the specific viscosity is the highest. Likewise as the ratio of actin to myosin in actomyosin differs a difference in the depression of viscosity is also observed. For example, when the content of actin in actomyosin is high, the depression of viscosity is large, while on the contrary, when the content of myosin is high, depression is small. Consequently, the degree of the depression of viscosity indicates the changes in the constitutional structure of myosin B.

Results on ATP-sensitivity is presented in Table II. As can be observed in the table, ATP-sensitivity of myosin B prepared from various kinds of meat was higher in AML than in AMS. This result was already suggested by one of the authors in a previous paper." Moreover, Yagi et al. reported that when myosin B was extracted for an extended period, ATP-sensitivity of resulting myosin B assumes a high value. ${ }^{3)}$ ATP-sensitivity of AMS prepared from rabbit and horse meat showed the highest value after 4 to 8 days of aging which decreased thereafter, and in case of AMS from veal and cow meat a decrease was observed only during the aging process. Consequently, ATP-sensitivity became lower with the progress of aging, and gradually approached a value almost uniform. It was

\footnotetext{
6) Harry L. Seagran, Food Res., 21, 505 (1956).

7) Szent-Györgyi, Muscular Contraction, 75, 152 (1951), Academic Press Inc., New York.
}

TABLE II ATP-SENSITIVITY OF MYOSIN B SOLUTION

\begin{tabular}{|c|c|c|c|}
\hline \multirow{2}{*}{$\begin{array}{c}\text { Kinds of animal } \\
\text { meats }\end{array}$} & \multirow{2}{*}{$\begin{array}{l}\text { Aging period } \\
\text { (Days) }\end{array}$} & \multicolumn{2}{|c|}{ ATP-sensitivity $(\%)$} \\
\hline & & AMS & AML \\
\hline \multirow{3}{*}{ Rabbit } & 0 & 26.5 & 148.3 \\
\hline & 4 & 132.5 & 112.5 \\
\hline & 8 & 5.0 & 97.6 \\
\hline \multirow{5}{*}{ Veal (Holstein Kind) } & 0 & 85.5 & 123.5 \\
\hline & 2 & 26.5 & 100.9 \\
\hline & 4 & 9.1 & 100.8 \\
\hline & 8 & 12.1 & 141.0 \\
\hline & 12 & 4.3 & 120.1 \\
\hline \multirow{5}{*}{$\begin{array}{l}\text { Veal (Japanese Black } \\
\text { Breed of Cattle) }\end{array}$} & 0 & 34.8 & 134.8 \\
\hline & 2 & 9.6 & 137.6 \\
\hline & 4 & 33.8 & 139.3 \\
\hline & 8 & 1.6 & 86.5 \\
\hline & 12 & 9.8 & 98.9 \\
\hline \multirow{4}{*}{ Cow meat } & 0 & 23.8 & 60.5 \\
\hline & 2 & 11.9 & 131.7 \\
\hline & 4 & 5.6 & 104.0 \\
\hline & 8 & 3.2 & 124.0 \\
\hline \multirow{4}{*}{ Horse meat } & 0 & 16.1 & 63.7 \\
\hline & 4 & 52.9 & 55.8 \\
\hline & 8 & 72.2 & 76.9 \\
\hline & 12 & 32.9 & 51.9 \\
\hline
\end{tabular}

reported that freshly prepared actomyosin readily dissociates to myosin and actin by the addition of ATP, but becomes difficult to dissociate when stored for a definite period.? Comparatively low values for ATP-sensitivity did not seem to be due to this cause; on account of their high ATP-sensitivity of AML from the same sample meats. From the results on ATP-sensitivity of AMS, it was indicated that the constitutional structure of AMS has been changed during aging, and the proportion of myosin in actomyosin becomes larger, namely the chemical nature of AMS approaches that of myosin. The same conclusion will be inferred from the results on salting out previously described.

ATPase : Changes of optimum pH of myo$\sin$ B-ATPase during the aging of meats. Some properties of ATPase of AMS and AML have already been reported in a previous paper..$^{2)}$ The enzymatic activities and the changes of optimum $\mathrm{pH}$ values of the two kinds of 
TABLE III

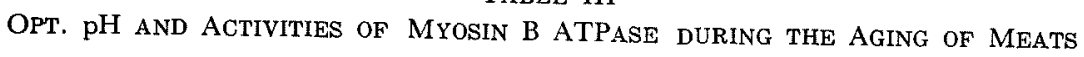

\begin{tabular}{|c|c|c|c|c|c|}
\hline Kinds of meats & $\begin{array}{l}\text { Aging period } \\
\text { (Days) }\end{array}$ & \multicolumn{2}{|c|}{$\begin{array}{l}\text { AMS-ATPase } \\
\text { opt. pH activity }\end{array}$} & \multicolumn{2}{|c|}{$\begin{array}{l}\text { AML-ATPase } \\
\text { opt. } \mathrm{pH} \text { activity }\end{array}$} \\
\hline \multirow{6}{*}{ Rabbit } & \multirow{2}{*}{0} & $\int 7.3$ & 38.3 & 7.3 & 36.4 \\
\hline & & $\{10.2$ & 69.7 & 10.2 & 90.5 \\
\hline & \multirow{2}{*}{4} & $\int 7.3$ & 41.5 & 7.3 & 43.1 \\
\hline & & l10.2 & 91.3 & 9.5 & 50.4 \\
\hline & \multirow{2}{*}{8} & $\{6.2$ & 17.9 & 6.2 & 22.0 \\
\hline & & $\{10.2$ & 12.3 & 10.2 & 29.8 \\
\hline \multirow{7}{*}{ Veal } & 0 & 7.1 & 27.0 & 6.7 & 21.4 \\
\hline & 2 & 7.1 & 13.4 & 6.7 & 26.5 \\
\hline & 4 & 6.7 & 14.7 & 6.7 & 23.1 \\
\hline & 8 & 6.7 & 17.9 & 6.7 & 31.5 \\
\hline & 12 & 6.7 & 16.1 & $6.2-6.7$ & 12.1 \\
\hline & 0 & 10.2 & 27.8 & & \\
\hline & 12 & 9.0 & 12.2 & & \\
\hline \multirow{4}{*}{ Horse meat } & 0 & 6.5 & 45.2 & 6.5 & 23.1 \\
\hline & 4 & - & - & 6.5 & 25.3 \\
\hline & 8 & 6.5 & 22.2 & 6.5 & 27.4 \\
\hline & 12 & 6.5 & 13.6 & 6.5 & 21.2 \\
\hline
\end{tabular}

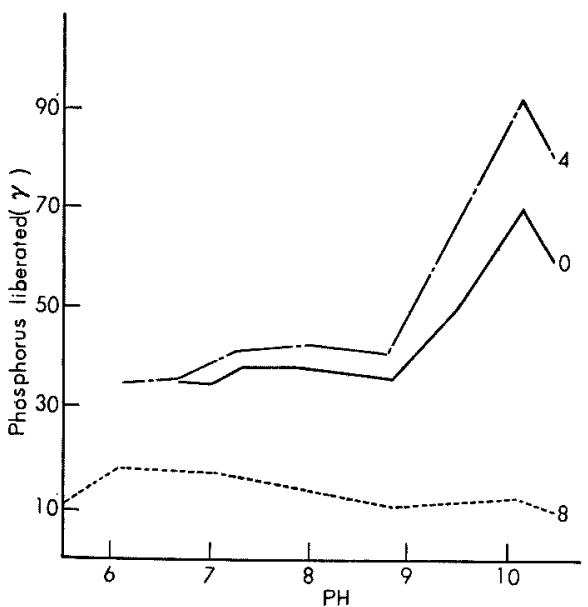

FIG. 3. Opt. pH of ATPase of Rabbit-AMS. Numbers in Fig. show days of aging.

ATPases during the aging of meats are presented in Table III and in Figs. 3 and 4.

Table III and Fig. 3 indicate that each ATPase of AMS and AML obtained from rabbit has two $\mathrm{pH}$ optima, one near the neutral point $(\mathrm{pH} 7.3)$, and the other in the alkaline region ( $\mathrm{pH}$ 10.2). After 8 days of aging, the optimum $\mathrm{pH}$ at 7.3 shifted to the

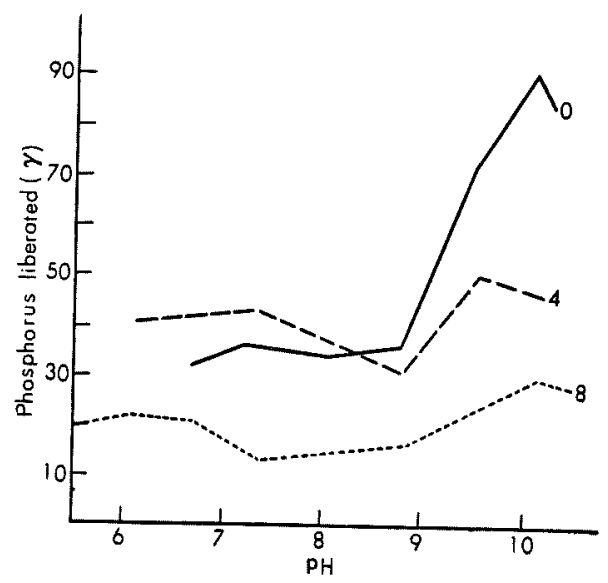

FIG. 4. Opt. pH of ATPase of Rabbit-AML. Numbers in Fig. show days of aging.

acidic side, i. e., $\mathrm{pH}$ 6.2. Also, in myosin $\mathrm{B}$ obtained from veal, the optimum $\mathrm{pH}$ value shifted to the acidic side in the same manner as in rabbit during the aging of meat, as it can be seen in Table III. Whereas in the case of myosin B from horse meat, no change in the optimum $\mathrm{pH}$ was observed during aging process (Table III). 
ATPase activity : As shown in Table III, ATPase activity of AMS and AML from rabbit was rather higher at the alkaline region than at the neutral $\mathrm{pH}$, but the difference of enzymatic activities at these two $\mathrm{pH}$ values almost diminished after 8 days of aging.

Changes of ATPase activities during aging are described as follows. At the neutral optimum $\mathrm{pH}$, little difference was recognized between the ATPases of AMS and AML. No remarkable changes of the activities were recognized for 4 days, but these activities decreased after 8 days of aging. At the alkaline optimum $\mathrm{pH}$, ATPase activities of AMS showed a maximum on the 4 th day of aging, while those of AML decreased with aging.

In Table III, results on ATPase activities of myosin $B$ from veal and horse meat are presented, but the experiments are confronted with activities at the neutral optimum $\mathrm{pH}$ value.

In the case of ATPase of AMS from veal, which differed from rabbit, difference was unrecognizable between enzymatic activities at both optimum $\mathrm{pH}$ values of 7.3 and 10.2.

Enzymatic activities of myosins from rabbit and veal (the same meat materials as were used to prepare myosin B) at the neutral optimum $\mathrm{pH}$ were 38.0 and $29.8(\gamma)$, respectively. Therefore, the ATPase-activity immediately after the slaughter seemed to be in the order: myosin $\geqslant A M S \geqslant A M L$. On the contrary, in the case of aged meats, enzymatic activity of AML was higher than that of AMS as previously reported. ${ }^{2)}$

As shown in Table III, enzymatic activity of veal myosin $B$ was the lowest among the myosin B which were obtained from three kinds of meats (rabbit, veal and horse meat).

\section{SUMMARY}

In order to decide whether or not, the chemical properties of myosin B extracted, isolated, and prepared from rabbit, veal, cow, and horse meat have been altered during aging, experiments were carried out on salting out, viscosity, ATP-sensitivity, and the ATPase of myosin B solution.

The results obtained are as follows:

(1) Salting out experiments showed that there is no difference of myosin $B$ to be observed in accordance with the kinds of animals tested. However, in the salting out procedure of AMS and AML solutions, the concentration of ammonium sulfate at the maximum precipitating region was found to be different. The concentration of ammonium sulfate in the salting out of myosin B was altered with the aging of meats; especially, in the case of AMS the maximum precipitating region seemed to approach that of myosin.

(2) Among all the kinds of animal meats tested, intrinsic viscosity of AML was higher than that of AMS, and intrinsic viscosity of myosin B was altered with the aging of meats. Intrinsic viscosity of the myosin B solution seemed to be different according to the kind of meat, i.e., that of myosin $\mathrm{B}$ from rabbit being high, while that obtained from cow meat was low.

(3) The ATP-sensitivity of AML was higher than that of AMS. The ATP-sensitivity of AMS became very low as aging proceeded. From this result, it was considered that AMS turns to assume a high ratio of myosin in the constitution of actomyosin, and comes to resemble myosin during aging.

(4) With the aging of meats, the optimum $\mathrm{pH}$ for ATPase of myosin B from rabbit and veal meat turned to the acidic side. At the neutral optimum $\mathrm{pH}$, enzymatic activity showed a tendency to decrease during aging. Immediately after slaughter, the activity of ATPase of AMS became higher than that of AML, but the inverse relation was maintained during aging process.

Immediately after slaughter, the optimum $\mathrm{pH}$ for enzymatic activity of AMS from rabbit were found to be 7.3 and 10.2, activity at $\mathrm{pH} 10.2$ being higher than that at $\mathrm{pH} 7.3$. However no difference was observed at both 
of these $\mathrm{pH}$ values in ATPase activity of AMS from veal immediately after slaughter.

Acknowledgements The authors wish to express their sincere thanks to Prof. R. Sasaki,
Prof. Y. Sakurai and Prof. M. Kandatsu for their interest in our work, and also to Dr. M. Nakamura for his kind advice.

[Bull. Agr. Chem. Soc. Japan, Vol. 22, No. 4, p. 255 261, 1958]

\title{
Effect of Gamma-Ray upon Food Microorganisms
}

\section{Studies Concerning Gamma-Ray Resistivity of Escherichia Coli}

\author{
By Wataru Watanabe \\ Department of Agricultural Chemistry, Tokyo University of Education, and \\ Niko Institute of Food, Niko Co., Ltd.
}

Received February 19, 1958.

\begin{abstract}
The author has carried out studies concerning the effects of different conditions on the gamma-ray resistivity of $E$. coli. This report deals with the influence of the heating procedure applied to irradiated samples after irradiation, the gamma-ray resistivities of strains stocked for long periods, and the influences of the addition of heat sterilized cells of the same strain and chemicals on mediums.
\end{abstract}

\section{INTRODUCTION}

In the sterilization of foods with ionizing radiation, destruction of nutrient components, color change, off-flavor and the decomposition of structure of the irradiated foodstuff used, result from extreme irradiation dose. Therefore, the minimum quantity of dose required to destroy the living cell of microorganisms should be investigated in order to minimize these unreasonable changes occurring in foodstuffs. This implies studies concerning the radio-resistivity of various types of microorganisms under different conditions and the comparison of the radio-resistivity of each microorganism.

Studies concerning the effect of post- and pre- treatments on bacterial inactivation have been investigated by many workers. BayneTones has conducted studies concerning the action of X-ray upon microorganisms at varied temperatures and has reported that the lethal action of X-rays, radioactive emanations and ultraviolet light are not affected by temperature, while various physical and chemical agents affect the lethal action of radiation to various degrees within a certain range. ${ }^{1,2), 8}$ Stapleton has conducted studied concerning the survival of an irradiated strain of $E$. coli with X-ray incubated at varied temperatures and has reported that the living cells of the irradiated strain are most survived in the medium, in case of incubation at $18^{\circ} \mathrm{C}_{\left.\left.0^{4}, 5\right\rangle, 6\right)}$

1) S. Bayne-Tones, and Van der Lingen, J.S. ; The bacterial action of ultraviolet light, Jobns Hopkins Hospital Bulletin, 34, 11-16, (1923)

2) F. I., Gates; A study of the bacterial action of ultraviolet light I and II, J. Gen. Pbysiol., 13, 231-248 and 249-260, (1929).

3) D. E. Lea., R. B. Haines and C. A. Coulson: The action of radiations on bacteria III, Roy. Soc. Proc. B., 123, 1-21, (1937).

4) G. E. Stapleton, D. Billen and A. Hollander: Recovery of $\mathrm{X}$-irradiated bacteria at suboptimal incubation temperature, $J$. Cellular. Comp. Physiol., 41, 345-358, (1953).

5) D. Billen, G. E. Stapleton and A. Hollander : The effect of $\mathrm{X}$-radiation on the respiration of E. coli, J. Bacteriol., 65, 131-

135, (1953). diation release of adenosinetriphosphate from $E$. coli $\mathrm{B} / \mathrm{s}$, Arch. Biochem. and Biophys., 43, 1-10, (1953). 